\title{
ÉTUDE EXPÉRIMENTALE DES COMPORTEMENTS DE CIVELLES D'ANGUILLA ANGUILLA L. INFLUENCE DE LA DENSITÉ ET DE LA DISPONIBILITÉ EN ABRIS
}

\author{
A. BARDONNET (1), C. RIGAUD (2), J. LABONNE (1)
}

(1) INRA UMR ECOBIOP, Unité de recherche en Hydrobiologie, quartier Ibarron, 64310 St Pée sur Nivelle, France.

Courriel : bardonne@st-pee.inra.fr, labonne@st-pee.inra.fr

(2) Cemagref, Département "Milieux Aquatiques », Unité Ecosystèmes estuariens et poissons migrateurs amphihalins, 50 Avenue de Verdun, Gazinet, 33612 Cestas cedex, France.

Courriel : christian.rigaud@bordeaux.cemagref.fr

Reçu le 10 octobre 2004

Accepté le 19 décembre 2005

Received October 07, 2004

Accepted December 19, 2005

\section{RÉSUMÉ}

Les comportements d'émigration et d'utilisation de l'espace développés par des civelles ont été observés dans des bacs et plans d'eau expérimentaux. Dans les bacs accueillant $55 \mathrm{ind} . / \mathrm{m}^{2}$ sans possibilité d'émigration, la densité d'abris végétaux a été contrôlée (densités d'abris basse, 2 tiges de végétation artificielle par bac ou haute, 9 tiges par bac) et le suivi a duré 2 mois et demi. Dans les plans d'eau ouverts, la densité de civelles introduites a été définie selon plusieurs modalités $\left(1,6-6-9\right.$ et $\left.12 \mathrm{ind} . / \mathrm{m}^{2}\right)$ et le suivi a duré 1 mois et demi. En bacs, après une période de 5 à 6 jours, les abris sont fortement recherchés pendant environ 6 semaines, les individus montrant alors une forte grégarité. Cette dernière disparaît ensuite, les abris sont alors moins recherchés et des comportements agressifs font leur apparition. En plans d'eau, les sorties concernent en moyenne $17 \%$ du stock initial (mini $=7 \%$, maxi $=35 \%$ ), interviennent rapidement et sont presque toutes dirigées vers l'amont. Leur intensité ne dépend pas de la densité initiale et apparaît plus importante dans les bassins sans herbier. Les tailles et poids moyens des émigrants sont plus importants que ceux du lot initial. Au terme du suivi, la croissance apparaît forte, hétérogène et associée à une faible survie dans les plans d'eau peu ou moyennement alevinés. Elle apparaît plus faible, homogène et associée à une bonne survie dans les bassins à forte densité initiale. Ces observations sont compatibles avec l'expression d'un comportement grégaire en condition de densité importante, comportement favorisant une croissance homogène, ayant pour conséquence de retarder l'apparition d'actes agressifs. Au contraire, dans des contextes de faible densité, l'expression rapide du potentiel de croissance de quelques individus favoriserait l'apparition d'actes agressifs.

Mots-clés : civelle, abri, sédentarisation, croissance, grégarité, agressivité, relation inter-individuelle. 


\title{
EXPERIMENTAL STUDY ON GLASS EEL BEHAVIOUR : INFLUENCE OF FISH DENSITY AND SHELTER AVAILABILITY
}

\begin{abstract}
Glass eel movements and shelter use were studied under experimental conditions in small indoor channels and in ponds. Shelters densities (2 stems of artificial plant, 9 stems) were controlled in indoor channels stocked with 55 ind. $/ \mathrm{m}^{2}$. Glass eel densities were controlled in ponds $\left(1,6-6-9\right.$ and 12 ind. $\left./ \mathrm{m}^{2}\right)$. Experiments lasted for two months and half in channels, and one month and a half in ponds. In indoor channels, glass eels highly looked for shelters after 5-6 days. During this phase, which lasted for 1 month and half, individuals were very gregarious. Thereafter, fish became aggressive and stopped using shelters. In ponds, emigration at the outlet was almost null. At the inlet, 3 phases were observed : the first one ( 15 days) with many captures, the second one (1 month) without any emigration and the third one, just before the end of the experiment, with few catches. The emigration rate was independent from initial densities, but increased in ponds with no hydrophyte development. The emigration at the inlet occurred for only a short period in the high-density ponds (first 5-6 days), whereas it lasted longer in the low-density ponds (15-20 days). After four weeks without captures, growth appeared high, heterogeneous and associated to low survival rates in the ponds with initial densities less than 10 ind./ $\mathrm{m}^{2}$. At higher densities, growth was lower, but homogeneous and associated with good survival rates. That suggests the development of agonistic behaviour or cannibalism in the lowest density ponds linked with a faster growth of some individuals. In high-density ponds, glass eels would exhibit a gregarious behaviour, leading to high survival and homogeneous but low growth. In these ponds the development of agonistic behaviour would be delayed possibly until fish would have reached a certain size and/or large interindividual differences in size have appeared.
\end{abstract}

Key words: glass eel, shelter, growth, movements, agonistic behaviour, territoriality, inter-individual relationships.

\section{INTRODUCTION}

L'anguille européenne (Anguilla anguilla) réalise des déplacements sur des distances considérables entre son aire de reproduction (Mer des Sargasses) et son aire de croissance ouest-européenne et méditerranéenne. Cette croissance s'effectue dans des milieux aussi divers que les zones côtières, les estuaires, les marais et étangs, les cours inférieurs des fleuves jusqu'aux ruisseaux de tête de bassin. Ce caractère très ubiquiste en termes d'utilisation du milieu est l'une des particularités de cette espèce rencontrée dans une gamme étendue de salinité et de température.

La phase de colonisation du bassin versant constitue une période clé débouchant sur une répartition des individus au sein des habitats qui déterminera en partie les caractéristiques du flux de géniteurs produits (PARSONS et al., 1977 ; VOLLESTAD et JONSSON, 1986 ; NAISMITH et KNIGHTS, 1993; JELLYMAN, 1997 ; JELLYMAN et CHISNALL, 1999 ; OLIVIERA, 2001).

Dans la zone soumise au flot, la civelle utilise majoritairement le courant de marée pour progresser vers l'amont (CREUTZBERG, 1961; GASCUEL, 1986 ; ELIE et ROCHARD, 1994), des phénomènes agrégatifs plus ou moins liés aux contraintes hydrologiques pouvant apparaître. Cette progression vers l'amont n'est pas systématique puisque certains individus se sédentarisent en estuaire salé (TZENG et al., 1997). Pour les individus franchissant la limite de marée dynamique, la nage active à contre-courant agrémentée parfois de phases d'escalade devient bien sûr obligatoire pour progresser vers l'amont. Cette transition comportementale entre l'estuaire et la zone fluviale a été 
peu étudiée jusqu'à présent. On peut noter en tous cas un décalage dans le temps entre l'arrivée significative d'individus dans la zone tidale amont et l'apparition des premiers phénomènes significatifs de migration active en amont de cette limite (voir synthèse de FEUNTEUN et al., 2003). L'influence de facteurs déclencheurs tels que la température de l'eau (WHITE et KNIGHT, 1997) est souvent évoquée pour expliquer ce délai. On notera enfin que la chute d'abondance de l'espèce se traduit depuis au moins deux décennies par la disparition des cordons remontant à contre-courant sur des distances parfois importantes (TESCH, 1977), cordons surtout composés d'individus en cours de pigmentation (VI A3 et plus) et au sein desquels la dépense d'énergie pour contrer le courant devait être largement diminuée par le développement de ce comportement agrégatif.

La répartition de l'espèce dans les zones dulçaquicoles des bassins versants pourrait résulter d'une diffusion des individus à partir du point source représenté par la limite de marée dynamique (SMOGOR et al., 1995 ; IBBOTSON et al., 2002). Une variabilité significative des comportements individuels (intensité et étendue des déplacements) est cependant souvent observée. FEUNTEUN et al. (2003) suggèrent ainsi l'existence, au cours de la première année de vie continentale, de deux types d'individus, les pionniers et les fondateurs. Les premiers auraient tendance à se déplacer très vite vers l'amont dès que les conditions le permettent (débit favorable, capacité physique...), tandis que les seconds s'établiraient dès que des conditions favorables apparaissent. Cette hypothèse de travail sous-entend l'existence chez ces très jeunes individus, de contingents aux caractéristiques particulières et fixes. D'autres auteurs (par exemple VAN BAALEN et HOCHBERG, 2001), à partir de travaux menés sur différentes espèces animales, plaident plutôt pour des histoires de vie résultant d'une succession de moments clés réclamant l'expression d'un comportement (le plus souvent « stay or leave ») lié au statut de l'individu à ce moment précis (état énergétique, état physiologique...). Ces deux hypothèses de travail sont en tous cas compatibles avec les résultats de FONTAINE et CALLAMAND (1941) révélant une activité thyroïdienne importante chez les individus présentant un rhéotropisme et un hydrotropisme exacerbés. Enfin, IBBOTSON et al. (2002) comme ELIE et ROCHARD (1994) soulignent le rôle (déclencheur, modérateur...) des contraintes de l'environnement (température, capacité d'accueil des milieux, compétition inter et intraspécifiques..) sur le comportement de migration.

Si quelques études ont révélé l'influence de la densité d'individus sur la croissance de l'anguille jaune (VOLLESTAD et JONSSON, 1988 ; DE LEO et GATTO, 1996), cette influence a été peu étudiée sur le stade civelle. Des suivis d'alevinages de civelles en petits plans d'eau clos (BELPAIRE et al., 1989, 1992 ; KLEIN-BRETELER, 1992 ; RIGAUD et MASSÉ, 2001) ont mis en évidence l'influence de la densité initiale sur la croissance moyenne individuelle au cours du premier été surtout à partir d'une densité de 1 ind./m². Elle pourrait traduire une compétition significative pour l'utilisation des sites (proies ou caches). En effet les civelles, tout comme les anguilles, ont besoin d'abris pendant leurs phases de repos (GLOVA et JELLYMAN, 2000) et le niveau de présence de ces abris pourrait donc représenter l'un des paramètres importants de la capacité d'accueil d'un milieu pour ces jeunes stades.

Deux expérimentations visant à préciser les phénomènes de compétition pour l'espace chez la civelle placée dans deux contextes expérimentaux différents ont été mises en place. La première, réalisée en milieu naturel contrôlé, vise à appréhender l'impact de la qualité du milieu et de la densité sur l'émigration et la croissance. La seconde, réalisée en milieu artificiel contrôlé, vise à préciser le rôle joué par les relations inter-individuelles dans l'utilisation des abris. Dans des petits plans d'eau, la densité en civelles a été manipulée (1 à 12 ind. $/ \mathrm{m}^{2}$ ) pour étudier son influence sur la croissance, l'émigration et la survie des civelles déversées. Dans les bacs, la densité d'abris a été manipulée pour étudier son influence sur la localisation et le comportement des civelles placées en forte densité (55 ind./ $\mathrm{m}^{2}$ ). 


\section{MATÉRIEL ET MÉTHODES}

\section{Suivis en petits plans d'eau}

9 plans d'eau ( $20 \mathrm{~m} \times 10 \mathrm{~m}$ ) alimentés en parallèle sur un canal d'amenée d'eau douce sont utilisés. La hauteur d'eau maximale y est de 0,65 $\mathrm{m}( \pm 0,05)$, la hauteur de vase de $0,10 \mathrm{~m}( \pm 0,05)$ avec un développement d'hydrophytes fixés (Potamogeton pectinatus) dans 6 des 9 bassins. Pendant les essais, un faible renouvellement en eau $(0,5 \mathrm{l} / \mathrm{s})$ est établi avec sortie possible des individus soit vers l'amont via une rampe-brosse, soit vers l'aval au fil de l'eau (surverse). Dans les deux cas, ils sont recueillis dans des pièges en tamis (maille $300 \mu \mathrm{m}$ ).

Les civelles (lot initial caractérisé sur un échantillon de 200 individus : $\mathrm{Lt}=64,3 \mathrm{~mm}$ $( \pm 0,8) ; P=0,22 \mathrm{~g}( \pm 0,03) ; 86 \%$ entre les stades VB et VIA ${ }_{2}$ de la classification de ELIE et al., 1982) ont été capturées par un pêcheur professionnel, dans la nuit du 14 au 15 mars dans l'estuaire de la Gironde, dans une zone de faible salinité (1 à $5 \%$ o). Leur lâcher réalisé (tableau I) selon 3 niveaux de densité (faible : 1,6 - moyen : 6 - fort : 9 et 12 ind./ $\mathrm{m}^{2}$ ) a eu lieu le 15 mars en fin d'après-midi $\left(J_{0}\right)$. La mortalité à court terme a été contrôlée en observant dans chaque plan d'eau 50 individus placés en cage-test pendant 24 heures. Chaque jour, les pièges amont et aval étaient relevés pour comptage et examen (taille, poids), les individus n'étant pas remis dans les bassins.

\section{Tableau I}

Répartition initiale des civelles par bassin, résultats des captures aux piègesbrosses (captures totales et captures sur les 15 premiers jours).

Table I

Glass-eels numbers stocked in each pond and number of catches at the outlet (total captures and captures of the first 15 days).

\begin{tabular}{|c|c|c|c|c|}
\hline Bassin & $\begin{array}{c}\text { Nombre } \\
\text { de civelles } \\
\text { déversées }\end{array}$ & $\begin{array}{c}\text { Densité } \\
\text { initiale } \\
\text { (Ind./m } \text { 2) }^{\text {) }}\end{array}$ & $\begin{array}{c}\text { Nombre de } \\
\text { remontées } \\
\text { (sur 15 jours/ } \\
\text { totales) }\end{array}$ & $\begin{array}{c}\text { Remontées } \\
\text { (sur 15 j/totales) } \\
\text { en \% du stock initial }\end{array}$ \\
\hline B 1 & 320 & 1,6 & $17 / 20$ & $5,3 / 6,2$ \\
\hline B 2 & 320 & 1,6 & $46 / 46$ & 14,4 \\
\hline B 3 & 320 & 1,6 & $112 / 112$ & $35,0 / 35,0$ \\
\hline B 4 & 1200 & 6 & $95 / 100$ & $7,9 / 8,3$ \\
\hline B 5 & 1200 & 6 & $133 / 133$ & 11,1 \\
\hline B 6 & 1200 & 6 & $295 / 342$ & $24,6 / 28,5$ \\
\hline B 7 & 1900 & 9 & $193 / 193$ & 10,1 \\
\hline B 8 & 2400 & 12 & $549 / 610$ & $22,9 / 25,4$ \\
\hline B 9 & 2400 & 12 & $381 / 382$ & 15,9 \\
\hline
\end{tabular}

Les caractéristiques morphologiques et les importances relatives des stades pigmentaires du lot initial déversé et des civelles piégées en amont (brosses) durant les 15 premiers jours ont été comparées respectivement par un test-t et par un test du $\chi^{2}$. L'influence des densités initiales ( 3 modalités) et de la présence ou non d'herbiers (2 modalités) sur les taux globaux de remontée sur 15 jours ont fait l'objet respectivement 
d'un test $\mathrm{H}$ de Kruskal-Wallis et d'un test $U$ de Mann-Whitney. Enfin, l'évolution sur les 15 premiers jours de la probabilité de sortie par les brosses a été traitée par modèle linéaire généralisé (procédure Genmod sous logiciel SAS). Le taux de sortie est modélisé par GLM de manière linéaire $\operatorname{logit}(T)=\log (T /(1-T))=a x+b$, où $x$ représente la période de capture entrée comme variable continue, a dépend du niveau de densité initiale (4 catégories) et b des trois variables catégorielles : densité initiale + herbier + vase (+ une constante correspondant au niveau de référence).

$A J_{47}$, une procédure standardisée de récupération des poissons restant dans les plans d'eau a été mise en œuvre. Durant un premier cycle de 24 heures, deux vidanges successives en après-midi ont été réalisées avec récupération des individus dans un long tamis filtrant le débit de sortie. Le bassin a ensuite été maintenu à sec hormis une petite rigole centrale reliant les 2 moines. Pendant la nuit suivante, un transit permanent d'eau dans cette rigole a permis de piéger en amont (brosse) comme en aval (filet). Après le relevé des pièges, les plans d'eau ont été remplis en début de matinée. Toutes les opérations ont alors être reprises à l'identique sur un second cycle de 24 heures.

Dans tous les bassins, l'épuisement des captures observé sur ces 2 cycles consécutifs a permis l'estimation du stock en place le plus probable selon la procédure utilisée en pêche électrique sur secteur fermé après passages successifs avec retrait (GERDEAUX, 1987). Tous les individus récupérés lors de ces vidanges finales ont été mesurés et pesés dans les bassins à faibles densités. Dans les bassins à moyenne et forte densités, ces mesures ont été réalisées dans chaque bassin sur un sous-échantillon de 300 individus prélevés aléatoirement. Ces données ont été comparées entre les 2 groupes de bassins présentant des densités finales supérieures et inférieures à 6 ind. $/ \mathrm{m}^{2}$ (test $\mathrm{U}$ de Mann-Whitney).

\section{Suivis en bacs}

Six bacs $(1,2 \mathrm{~m} \times 0,3 \mathrm{~m})$ à fond nu sont alimentés en eau en parallèle par une dérivation de la rivière Nivelle $\left(1^{\circ} 35 \mathrm{~W}, 43^{\circ} 20 \mathrm{~N}\right)$. Les abris sont constitués de végétaux artificiels ancrés sur le fond et s'élevant en pleine eau (type élodée, ie une tige simple comportant tous les $\mathrm{cm}$ environ des feuilles de $1,5 \mathrm{~cm}$ disposées en couronne). Quatre longueurs de tiges sont utilisées $(10$ à $37 \mathrm{~cm}$ ) à deux niveaux de densité : faible pour les bacs 2, 4 et 6 (2 tiges par bac) ; forte pour les bacs 1, 3 et 5 : (9 tiges). Température, turbidité, conductivité et pression atmosphérique sont enregistrées en continu (sonde multi-paramètres).

Les civelles ont été pêchées à Urt (25 km en amont de l'embouchure de l'Adour) en février et stockées en bac $\left(T=12^{\circ} \mathrm{C}\right)$. Fin Mars $\left(90 \%\right.$ de $\mathrm{VIA}_{0}-\mathrm{VIA} \mathrm{A}_{1}-\mathrm{VIA} \mathrm{A}_{2}$, selon les stades pigmentaires décrits par ELIE et al., 1982) chaque bac reçoit 20 de ces civelles (densité de 55 ind $/ \mathrm{m}^{2}$ ). Environ 500 civelles sont stabulées sur eau de Nivelle pour pouvoir compenser progressivement les mortalités enregistrées dans les bacs.

Une pâte alimentaire est distribuée une fois par jour sur une mangeoire amovible. Les nettoyages des bacs sont réalisés tous les 10 jours. A partir du 29/04, des bains de vert de malachite et de formol sont réalisés tous les 3 jours pour enrayer le développement de l'icthyophtiriose diagnostiquée, des traitements complémentaires étant effectués les 14/05 et 22/05.

Les bacs sont entourés d'un cache permettant la circulation dans la pièce sans être vu des civelles tout en autorisant leur observation grâce à des « fenêtres » (3 fenêtres par bac). Les civelles ont été installées le 25 mars et suivies pendant 2 mois et 10 jours. Leur localisation (repérage sur une carte du bac) et leur comportement (4 catégories : A (sur abri), B (au bas de l'abri), C (posées sur le fond) et D (en nage) sont relevés 2 fois par jour (9 h et 16 h), à raison de 4 à 5 jours chaque semaine. Des relevés de nuit ont été réalisés ponctuellement pour essayer d'évaluer aussi le comportement nocturne. Cependant, 
l'éclairement à la lampe même de faible intensité semblait avoir un effet perturbateur. Comme par ailleurs la distribution spatiale des civelles ne semblait pas différer entre le jour et la nuit, le suivi nocturne n'a pas été retenu. Afin de limiter les interventions dans les bacs, les mortalités ont été relevées et les mortes remplacées à un rythme hebdomadaire.

Les données sont traitées par un modèle linéaire généralisé avec comme variable dépendante le score (nombre de civelles observées par catégorie « comportementale »), les variables explicatives étant la modalité de comportement $(A, B, C, D)$ et la densité d'abris ( $1=9$ abris ; 2 = 2 abris).

Les scores peuvent être considérés comme la somme de tirages aléatoirement indépendants. Le modèle linéaire généralisé sera donc ajusté à une distribution poissonienne. L'effet des variables explicatives sera testé en réalisant une ANOVA sur la déviance expliquée par chaque variable. La qualité de l'ajustement du modèle sera évaluée en analysant les résidus.

\section{RÉSULTATS}

\section{Observations en petits plans d'eau}

Relevées en fin de matinée, les températures du canal d'amenée et des 9 bassins ont oscillé entre 13 et $15,5^{\circ} \mathrm{C}$, les $\mathrm{pH}$ étant compris entre 7 et 8 et les teneurs en oxygène dissous entre 9 et 12 mg/l (90 à $115 \%$ de saturation).

\section{Effectifs et cinétique de sortie des bassins}

En moyenne par bassin, 17,2 \% ( \pm 10$)$ des civelles introduites ont été reprises sur les brosses amont (Tableau I). La sortie vers l'aval au fil de l'eau n'a quasiment jamais été observée puisque, sur l'ensemble du suivi, seules 10 civelles ont été retrouvées dans le piège aval d'un bassin à forte densité initiale, 2 jours avant la vidange finale.

Globalement, $67 \%$ des civelles capturées à l'amont l'ont été dans les 3 premiers jours et $93 \%$ dans les 15 premiers jours. Après cette phase initiale, une quasi-absence de remontée est notée pendant un mois, une légère reprise n'étant observée qu'entre $J_{44}$ et $J_{47}$ (Figure 1). L'absence d'herbiers influence significativement le taux global de remontée sur les 15 premiers jours (Test $U$ unilatéral, $\alpha=0,05, p=0,01$ ). Par contre, ni la densité initiale, ni le niveau de vase du bassin n'ont d'effet significatif sur ce taux global (Figure 2).

L'analyse GLM de l'évolution de la probabilité de sortie par les brosses sur les 15 premiers jours précise un peu ces éléments (Figure 3). Une décroissance significative sur cette période est confirmée dans tous les bassins avec deux aspects à prendre en compte, le niveau initial de probabilité de sortie (résultats de la première période de 3 jours) et la pente ultérieure de décroissance de cette probabilité. L'absence d'herbier a un impact hautement significatif sur le niveau initial de probabilité de sortie et ce, quelle que soit la densité et le niveau d'envasement concernés. En ce qui concerne l'évolution ultérieure de la probabilité de sortie, il apparaît un effet significatif de la densité initiale d'individus, les 2 premières modalités de densité (1,6 et 6 ind. $\left./ \mathrm{m}^{2}\right)$ se caractérisant dans tous les cas par une décroissance moins rapide de cette probabilité sur les 4 périodes d'observation.

Les individus piégés lors de cette phase initiale ont une longueur et un poids moyens $\left(L_{\text {moy }}=66,1 \mathrm{~mm}( \pm 1,3)\right.$ et $\left.P_{\text {moy }}=0,25 \mathrm{~g}( \pm 0,05)\right)$ significativement plus élevés que ceux observés sur le lot initial (test-t unilatéral, $p<0,001$ ). Par contre, la distribution des stades pigmentaires n'est pas significativement différente de celle du lot initial $\left(\chi^{2}\right.$, $\mathrm{ddl}=5, \mathrm{p}<0,05)$. 


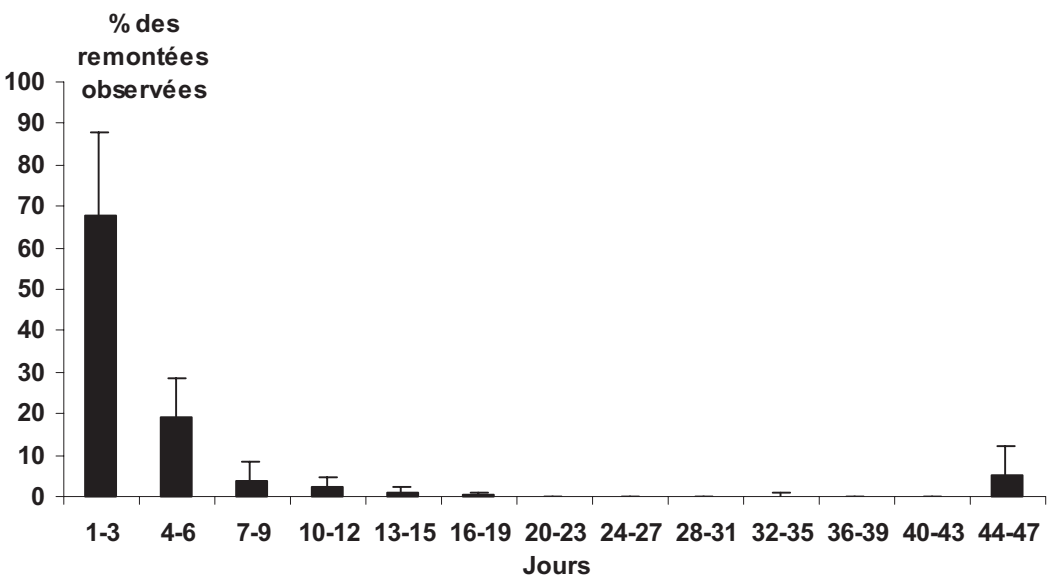

Figure 1

Evolution des captures moyennes observées aux brosses amont par période de 3 jours, captures exprimées en pourcentages du stock initial déversé.

Figure 1

Evolution of the mean number of catches at the outlets by 3 days-period.

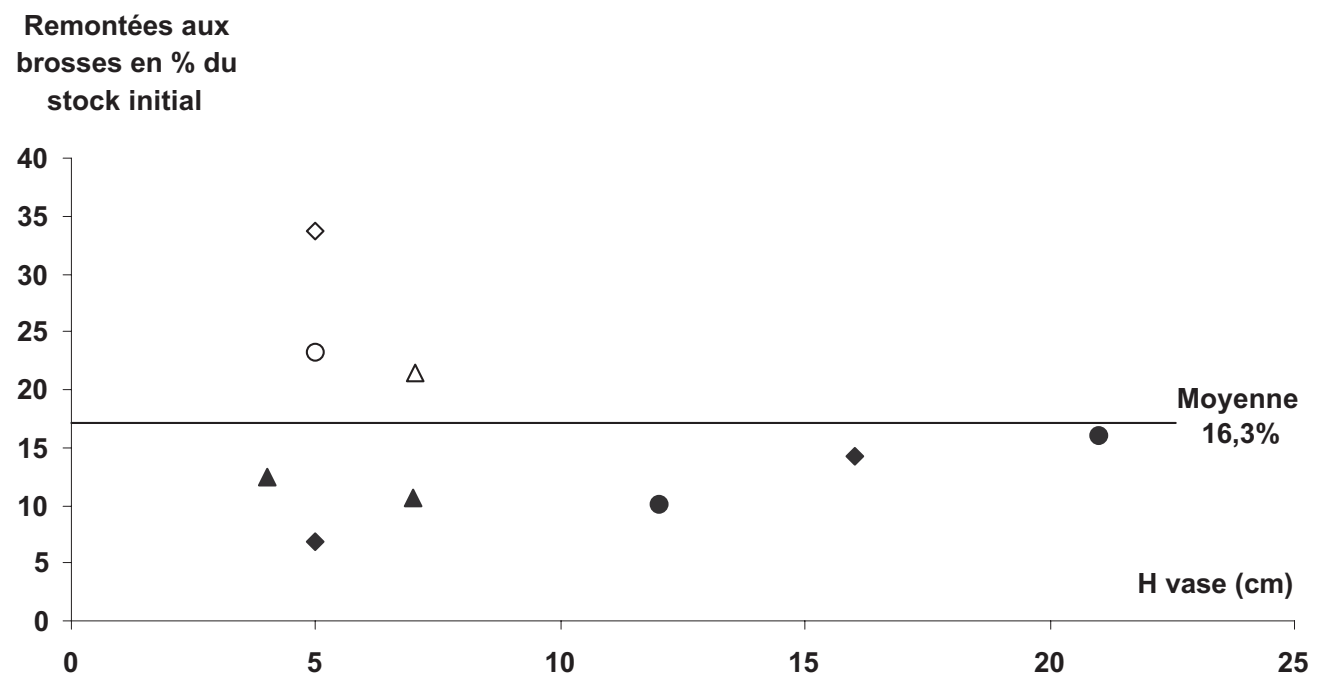

Figure 2

Taux global de remontée aux brosses sur les 15 premiers jours exprimés en pourcentage des stocks initiaux visualisés en fonction de la hauteur de vase et de la présence d'hydrophytes dans les bassins. Bassins à faible densité initiale (losanges), moyenne densité (triangles) et forte densité (cercles). Les symboles ouverts représentent les bassins sans végétation fixée.

\section{Figure 2}

Total captures at the outlets during the first 15 days (expressed in terms of percentages of the initial stocking densities) according to mud levels and hydrophytes abundances observed in the ponds. Low density ponds (diamonds), mean density ponds (triangles) and high-density ponds (circles). Open symbols represent the ponds without hydrophytes. 

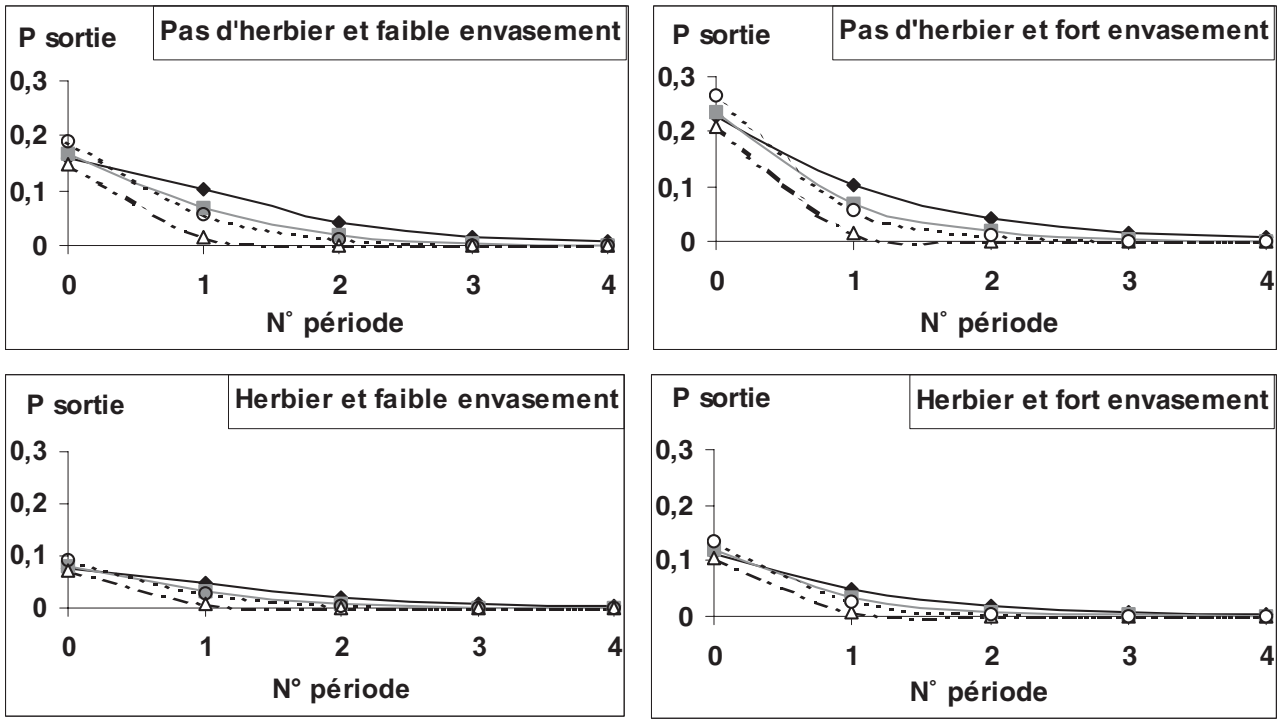

\begin{tabular}{|c|c|c|c|}
\hline \multicolumn{4}{|c|}{$\begin{array}{c}\text { Logit (Proba de remontée) }= \\
\text { constante + «période * densité " + « densité " + « herbier " + « vase " }\end{array}$} \\
\hline \multicolumn{2}{|c|}{ Lien : logit } & \multicolumn{2}{|c|}{ Distribution : binomiale } \\
\hline & $\begin{array}{l}\text { Significativité des effets } \\
\text { Test } \chi^{2}\end{array}$ & & Coefficients \\
\hline Constante & $<0,0001$ & & $-1,845$ \\
\hline \multirow[t]{4}{*}{ Période $\mathrm{x}$ densité } & $<0,0001$ & 1,6 & $-0,9445$ \\
\hline & & 6 & $-1,414$ \\
\hline & & 9 & $-2,734$ \\
\hline & & 12 & $-1,771$ \\
\hline \multirow[t]{4}{*}{ Densité initiale } & $<0,0001$ & 1,6 & $-0,203$ \\
\hline & & 6 & $-0,146$ \\
\hline & & 9 & $-0,295$ \\
\hline & & 12 & 0 \\
\hline \multirow[t]{2}{*}{ Herbier } & 0,0001 & Présence significative & $+0,819$ \\
\hline & & Absence & 0 \\
\hline \multirow[t]{2}{*}{ Vase } & 0,0001 & Faible envasement & $-0,426$ \\
\hline & & Fort envasement & 0 \\
\hline
\end{tabular}

Figure 3

Traitement par modèle linéaire généralisé de la probabilité de sortie d'un individu par les brosses sur les 15 premiers jours. 4 variables testées : densité initiale (12 ind. $/ \mathrm{m}^{2}$ : cercles ouverts; 9 ind. $/ \mathrm{m}^{2}$ : triangles ouverts ; 6 ind. $/ \mathrm{m}^{2}$ : carrés gris : ; 1,6 ind. $/ \mathrm{m}^{2}$ : losanges noirs) - croisement de la densité initiale et du jour (5 périodes de 3 jours) - présence d'herbier ( 2 modalités) - niveau d'envasement (2 modalités).

Figure 3

GLM analysis of the individual probability of capture at the outlet during the first 15 days. 4 tested variables : Initial density $\left(12\right.$ ind. $/ \mathrm{m}^{2}$ : open circles; 9 ind. $/ \mathrm{m}^{2}$ :

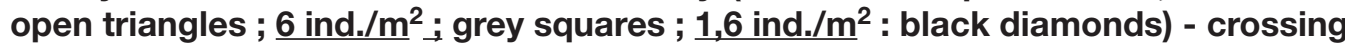
"density x Time (5 periods of 3 days)" - presence of hydrophytes (2 levels) - mud level (2 levels). 
Lors des 4 derniers jours, une très faible reprise des remontées aux brosses (115 captures, soit 6,2\% des remontées initiales) a été observée dans 5 des 9 bassins. 60 remontées ont ainsi été notées dans les 2 bassins à forte densité finale (soit 1,8 \% du stock récupéré 4 jours plus tard par vidange dans ces bassins), 3 dans un bassin à faible densité finale $(4,1 \%$ du stock final dans ce bassin) et 52 dans 2 bassins à densité finale intermédiaire $(5,3 \%$ du stock final de ces bassins).

\section{Croissance des individus}

Aux captures obtenues lors de la vidange finale d'un bassin ont été rajoutées les rares remontées aux brosses des 4 derniers jours. L'effectif et la biomasse des individus présents dans le bassin en fin d'expérimentation (Df et Bf) ont ainsi été reconstitués. L'effectif initial et la biomasse initiale déversés dans chaque bassin ont été quant à eux corrigés en retirant l'effectif et la biomasse sortis aux brosses pendant les 15 premiers jours d'expérimentation. On obtient ainsi pour chaque bassin la densité et la biomasse initiales effectives (Die et Bie) qui représentent au mieux les conditions de départ.

La biomasse finale calculée dans un bassin est linéairement (facteur 1,43) et significativement liée à la biomasse initiale effective de ce bassin (Figure 4). Cette biomasse finale résulte de la survie et de la croissance pondérale individuelle :

- la densité finale calculée apparaît positivement mais non linéairement liée à la densité initiale effective (Figure 5). On observe une tendance à l'amélioration du taux de survie avec l'augmentation de la densité initiale effective,

- les 2 bassins présentant les plus fortes densités finales correspondent aussi à des poids moyens individuels (Figure 6) significativement plus faibles et plus homogènes (faibles coefficients de variation) que ceux observés dans les 7 autres bassins (test $U$, unilatéral, $\alpha=0,05 ; p=0,028)$. Par contre, les tailles observées ne sont pas significativement différentes entre ces 2 groupes de bassins.

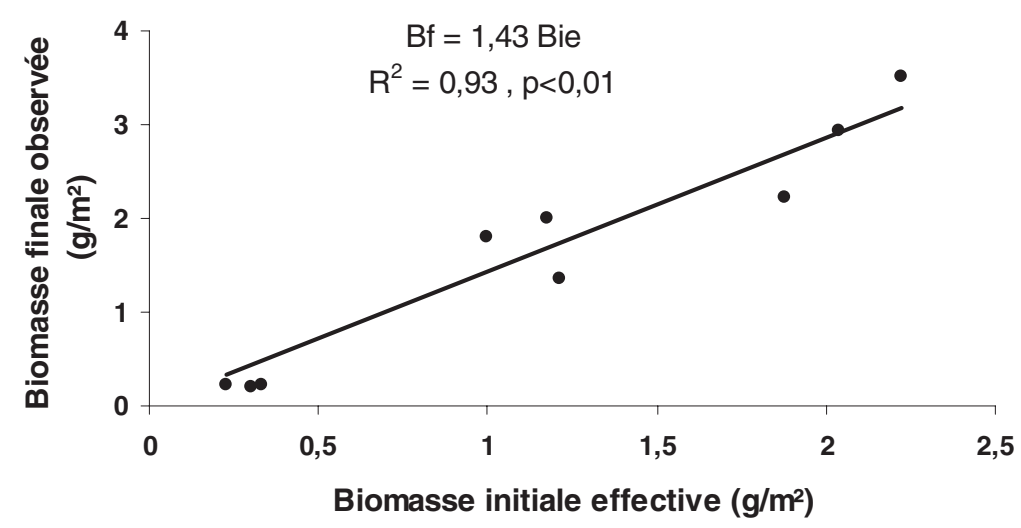

Figure 4

Biomasse finale calculée $\left(\mathrm{g} / \mathrm{m}^{2}\right)$ en fonction de la biomasse initiale effective (biomasse déversée - biomasse des remontées sur 15 jours).

\section{Figure 4}

Calculated final biomass $\left(\mathrm{g} / \mathrm{m}^{2}\right)$ according to the effective initial biomass (stocked biomass minus biomass caught at the outlets during the first 15 days). 


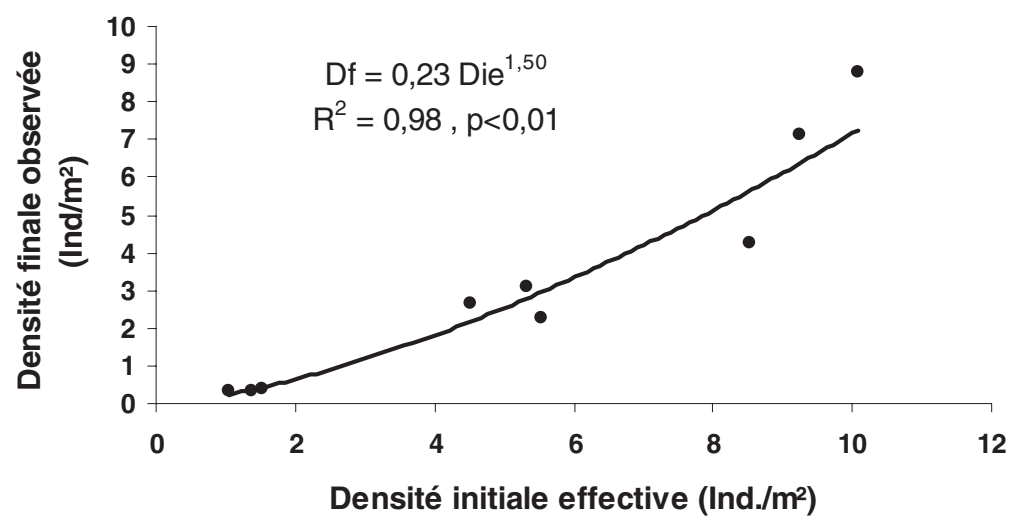

\section{Figure 5}

Relation entre les densités finales calculées et les densités initiales effectives (nombre d'individus déversés, - nombre d'individus capturés aux brosses sur les 15 premiers jours).

\section{Figure 5}

Relationship between the calculated final densities and the effective initial densities (initial stocked density minus the outlet catches of the first 15 days).

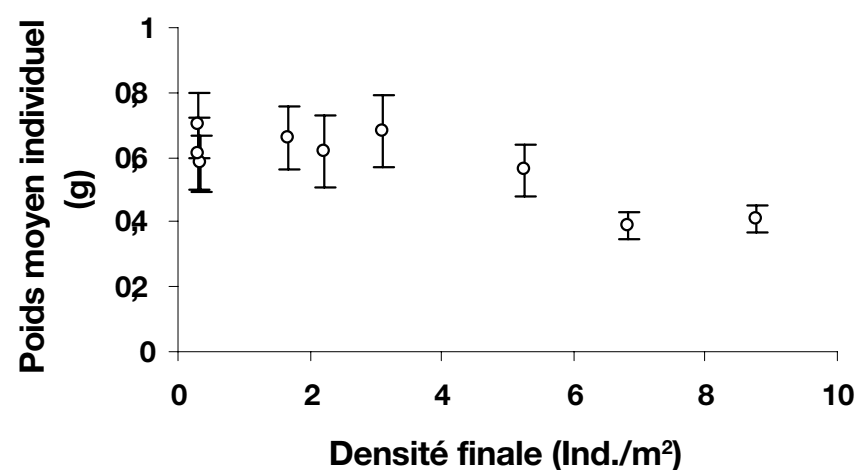

\section{Figure 6}

Poids moyens individuels à $\mathrm{J}_{47}$ en fonction des densités finales calculées.

\section{Figure 6}

Mean individual weight at $D_{47}$ according to the calculated final densities.

\section{Observations en bacs}

\section{Mortalité et paramètres environnementaux}

Des mortalités importantes sont survenues dans le bac de stabulation. Dans les bacs expérimentaux, les mortalités ont aussi été marquées avec 17, 12 et 11 mortes au total dans les bacs à forte densité d'abris et 20,17 et 15 dans les bacs à faible densité d'abris. La turbidité est restée faible en mars et en avril (0 à 25 NTU). Entre mai et juin, 3 crues de la Nivelle ont augmenté la turbidité jusqu'à plus de 100 NTU, mais elles ont été assez courtes (moins de 3 jours). La température a varié entre 10 et $18^{\circ} \mathrm{C}$, avec une moyenne aux alentours de $12,5^{\circ} \mathrm{C}$ en mars-avril. A partir de la deuxième semaine d'avril et jusqu'à mi-mai elles s'établissent autour de $14^{\circ} \mathrm{C}$, à l'exception d'une dizaine de jours 
plus frais $\left(12^{\circ} \mathrm{C}\right)$ début mai. Les températures continuent ensuite leur progression et varient entre $14^{\circ}$ et $18{ }^{\circ} \mathrm{C}$.

\section{Modèle Linéaire Généralisé : effet abris et comportement}

Les résultats de I'ANOVA indiquent qu'il n'y a aucun effet « densité d'abri » (Tableau II, $p=0,2)$. En effet, les scores prédits en fonction de la variable « densité d'abri » sont très comparables (Figure 7A). Les pourcentages de civelles sur abri ne sont pas significativement différents quelle que soit la densité d'abris.

Si l'effet abri est peu marqué, par contre, l'effet «catégorie comportementale » est très significatif (Tableau II, $p<0.0001$ ) : la distribution des scores n'est donc pas homogène entre les classes de comportement. Les scores prédits dans chacune des 4 modalités de la variable "comportement » diffèrent en effet de façon importante (Figure 7B). La modalité A (anguille posée sur abri) obtient le meilleur score, tandis que les civelles au bas de l'abri ou en nage sont peu nombreuses (modalités B et D). Enfin, les civelles posées sur le fond (catégorie $\mathrm{C}$ ) ont un score prédit intermédiaire. Les civelles ont donc fortement utilisé les abris de végétation artificielle. La position «posée sur le fond » a été vue relativement souvent comparée au nombre de civelles vues en nage ou au bas de l'abri.

L'interaction entre les deux variables explicatives apparaît aussi significative (Tableau II, $\mathrm{p}<0.0001$ ) : la distribution des scores selon les classes comportementales est dépendante de la densité d'abris. En effet, on observe une augmentation du nombre de civelles posées sur le fond (catégorie C) quand la densité d'abris diminue. L'impact de la densité d'abri est par contre peu marqué pour les autres comportements (Figure 7C).

L'analyse des résidus montre que la quantité de déviance résiduelle est importante. En théorie si la déviance résiduelle ne contient que de la variabilité aléatoire, elle suit une loi de $\chi 2$ à $\mathrm{n}$ ddl, $\mathrm{n}$ étant le nombre de degrés de liberté résiduels (BURNHAM et ANDERSON 1998). Ceci revient à considérer que le rapport (déviance résiduelle/ $\chi 2$ à $\mathrm{n}$ ddl) tend vers 1 . Or, il est ici supérieur à 2. Cela est sans doute dû pour partie à la distribution des scores qui est très étalée et au nombre important de scores nuls, mais il est aussi probable qu'un autre facteur, non pris en compte dans cette analyse, structure les données. Si on distribue les résidus en fonction du temps, on remarque qu'ils atteignent des valeurs extrêmes essentiellement en début et en fin de période (Figure 8).

\section{Tableau II}

Résultats de l'ANOVA à 2 facteurs réalisée sur les scores de déviance (mesure de l'ajustement aux données).

Table II

Results of two-ways ANOVA conducted on deviance scores (measure of fitting to data).

\begin{tabular}{|l|c|c|c|c|c|}
\hline & $\begin{array}{c}\text { Degrés } \\
\text { de liberté }\end{array}$ & $\begin{array}{c}\text { Déviance } \\
\text { expliquée }\end{array}$ & $\begin{array}{c}\text { Degrés de liberté } \\
\text { des résidus }\end{array}$ & $\begin{array}{c}\text { Déviance } \\
\text { résiduelle }\end{array}$ & $\begin{array}{c}\text { Probabilité } \\
(>\mid \text { Chi } \mid\end{array}$ \\
\hline Total & & & 2183 & 10849.2 & \\
\hline Facteur Abri & 1 & 1.5 & 2182 & 10847.7 & 0.2 \\
\hline $\begin{array}{l}\text { Facteur } \\
\text { Comportement }\end{array}$ & 3 & 5844.4 & 2179 & 5003.3 & $<0.0001$ \\
\hline $\begin{array}{l}\text { Interaction } \\
\text { Abri : comportement }\end{array}$ & 3 & 49.5 & 2176 & 4953.8 & $<0.0001$ \\
\hline
\end{tabular}


A

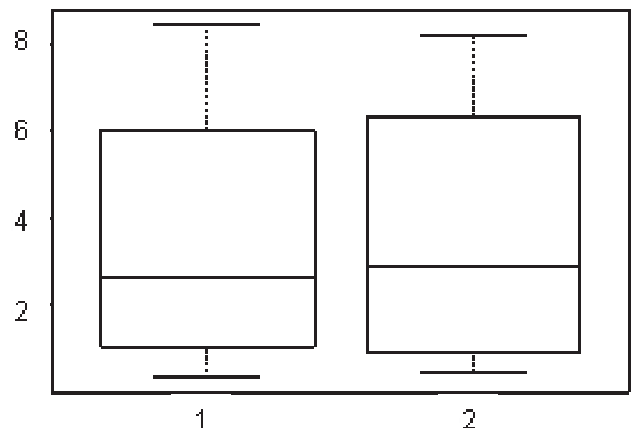

C

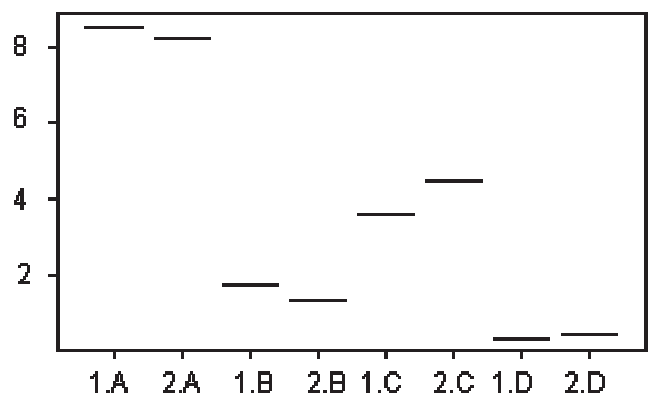

B

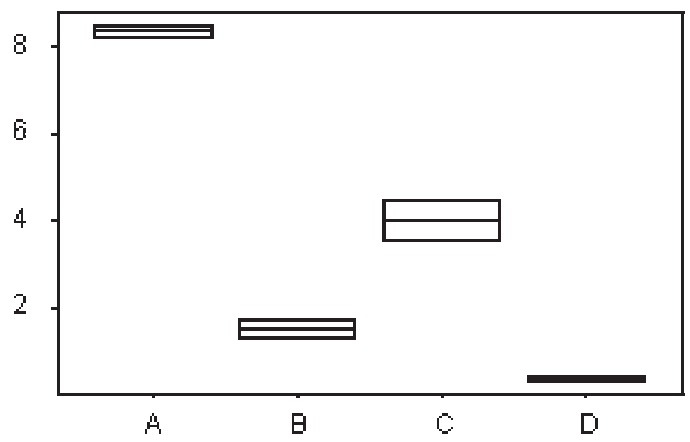

Figure 7

Représentation des scores prédits (nombre d'individus) en fonction :

A - de la densité d'abris (1 = 9 abris ; 2 = 2 abris)

B - du comportement ( $A=$ anguille sur abri ; $B=$ anguille au bas de l'abri ;

$C=$ anguille posée sur le fond ; $D=$ anguille en nage)

C - de la modalité abri (chiffre) et de la modalité comportement (lettre).

$1 . A=9$ abris, anguille sur abri

1.C $=9$ abris, anguille posée

2.A $=3$ abris, anguille sur abri

2.C $=3$ abris, anguille posée

1.B $=9$ abris, anguille au bas de l'abri

$1 . D=9$ abris, anguille en nage

2.B $=3$ abris, anguille au bas de l'abri

2.D = 3 abris, anguille en nage

Figure 7

Values of the predicted scores (number of individuals) according to :

A - shelters density ( $1=9$ shelters $; 2=2$ shelters)

$B$ - fish behaviour $(A=$ eel on shelter ; $B=$ eel at the foot of the shelter ; $C=$ eel on the channel bottom ; $D=$ eel swimming)

$C$ - shelters (number) and behaviour (letter).

$1 . A=9$ shelters, eel on shelter

2.A $=3$ shelters, eel on shelter

1.C $=9$ shelters, eel on the bottom

$1 . B=9$ shelters, eel at the foot of the shelter $1 . D=9$ shelters, eel swimming

2.B $=3$ shelters, eel at the foot of the shelter $2 . D=3$ shelters, eel swimming

Si on examine ensuite cette distribution pour chaque catégorie comportementale, on remarque qu'il y a une structuration dans le temps en opposition de phase pour les deux catégories $A$ et $C$ (Figure 9). C'est à dire que le nombre de civelles sur les abris et posés sur le fond varie au cours de la période de suivi, et que les changements s'opèrent essentiellement entre ces deux catégories comportementales. Elles sont aussi les plus souvent observées (Figure 7B), les catégories au pied de l'abri et en nage étant peu souvent observées. Le retour aux données montre que les civelles en début de période 


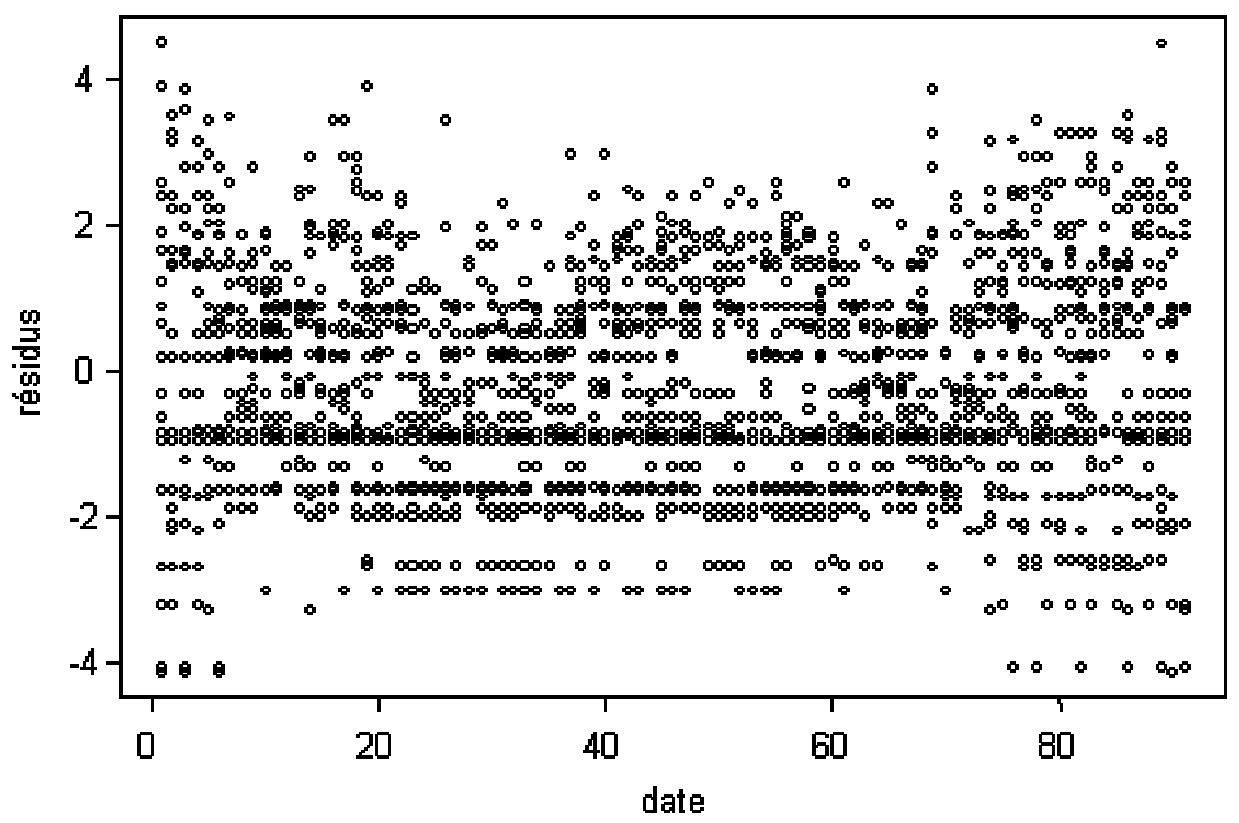

Figure 8

Distribution des résidus du Modèle Linéaire Généralisé en fonction du temps.

Figure 8

Distribution of residuals of the Generalized Linear Model according to time.

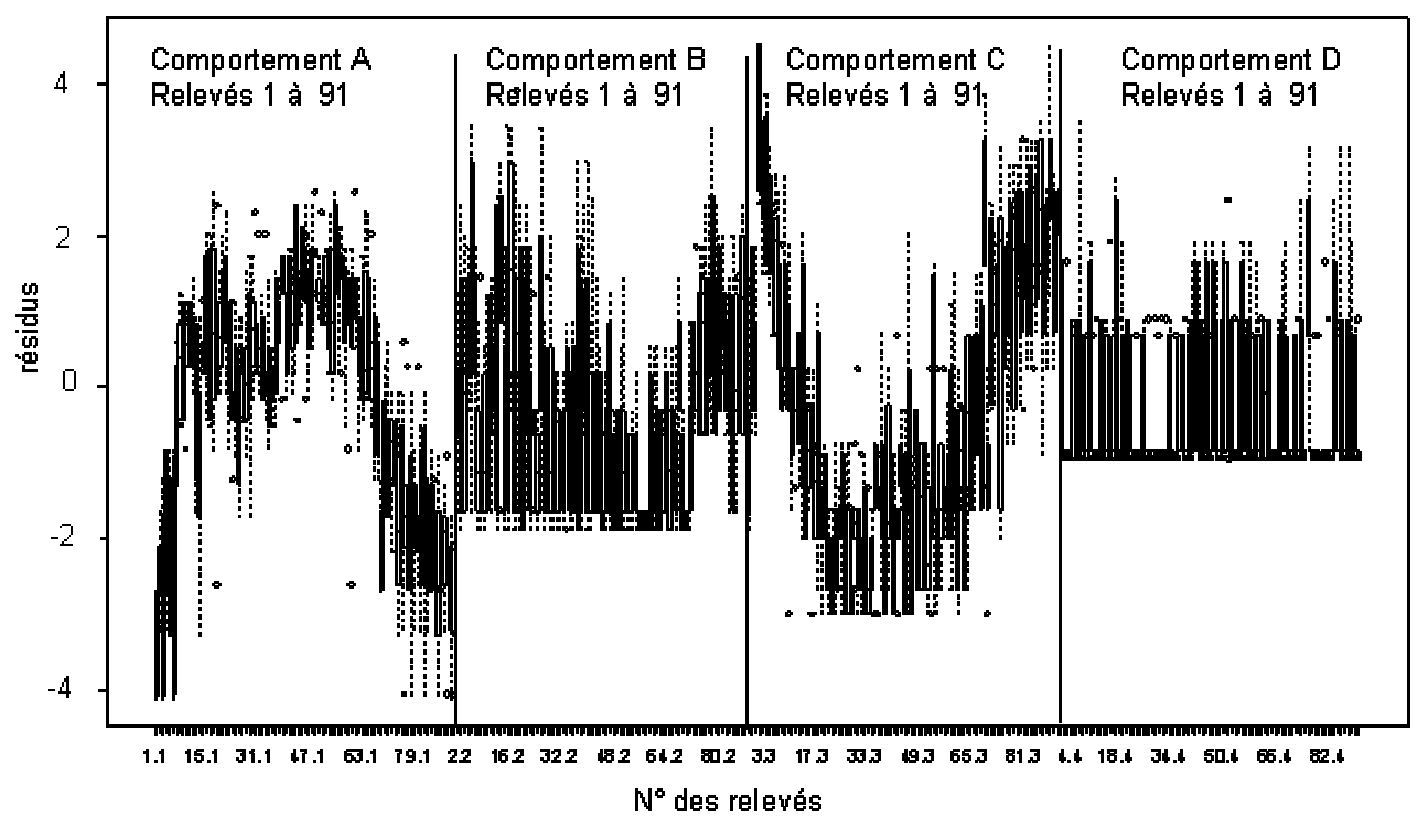

Figure 9

Distribution des résidus en fonction des modalités comportementales et du temps.

Figure 9

Distribution of residuals according to behaviour and time. 
d'observation sont majoritairement posées sur le fond. Elles se regroupent ensuite sur les abris, qu'elles quittent en fin de période d'observation. La première période dure entre 4 et 8 jours, alors que la seconde période au cours de laquelle les anguilles se regroupent sur les abris où elles forment des «nœuds de civelles » est beaucoup plus longue (environ 1 mois et demi). Au cours de la troisième période de suivi, les anguilles sont à nouveau en majorité posées sur le fond et de nombreux comportements agressifs apparaissent, mais ils n'ont pas été quantifiés.

\section{DISCUSSION}

\section{Utilisation des abris}

En bacs expérimentaux, les expériences de GLOVA (2001) avaient montré une bonne utilisation des macrophytes et du substrat de galets par les juvéniles d'anguille. L'avantage expérimental de l'abri végétal par rapport au substrat est que les animaux restent visibles. Tous les animaux ne sont cependant pas repérés à chaque relevé car il devient difficile de les compter lorsqu'ils se regroupent et l'observateur n'a qu'une vision partielle du corps des civelles situées au bas des abris. Cette catégorie a donc pu être sous estimée dans nos suivis. Ceci pourrait expliquer l'interaction entre la densité d'abris et le comportement observé en bac (Tableau II). En effet, avec 9 abris, les sites de cache disponibles au bas des tiges sont plus nombreux. Le nombre estimé de civelles en nage ou posées sur le fond est par contre certainement très proche de la réalité.

Dans cette expérience, l'utilisation des abris est comparable quelle que soit la densité en abris comme GLOVA (2002) l'avait déjà noté chez Anguilla australis. Les civelles se regroupent sur une ou plusieurs tiges, et forment des rassemblements ressemblant à des «nœuds ». Cette grégarité avait déjà été notée par TESCH (1977), puis par DOU et TSUKAMOTO (2003) qui observent que les civelles d'Anguilla japonica se mettent à plusieurs dans le même «tube abri », même si d'autres tubes sont libres. Cette phase de grégarité a duré, dans notre contexte d'expérimentation, jusqu'à la mi-mai environ, soit pendant 6 semaines. A partir de ce moment, on observe une diminution du nombre de civelles sur les abris au profit des civelles posées sur le fond.

Dans l'expérience en plan d'eau, au vu des taux d'émigration de certains bassins, la présence de macrophytes semble représenter un facteur important de la capacité d'accueil, au moins pendant la phase suivant la mise à l'eau. L'affinité de l'anguille pour les végétaux a aussi été signalée chez les petites anguilles en marais (LAFFAILLE et al., 2004). La vase en phase printanière et estivale ne semble pas par contre constituer un abri très recherché comme l'ont déjà constaté expérimentalement LECOMTE-FINIGER et PRODON (1979) et plus récemment SILBERSCHNEIDER et al. (2004).

\section{Survie et croissance}

Les observations de survie et de croissance au terme des vidanges finales des plans d'eau ont permis de visualiser le devenir des individus n'ayant pas opté pour l'émigration lors des 15 premiers jours. La mise en évidence d'une biomasse finale augmentant de façon linéaire avec la biomasse initiale effective semble traduire le caractère non saturé de la capacité d'accueil des bassins. Cependant, la différence significative dans les poids moyens observés et surtout l'homogénéité de ces poids dans les 2 bassins à forte densité initiale d'une part et dans les autres bassins d'autre part impose une interprétation plus nuancée. Ainsi dans les contextes de forte densité initiale, les individus à bon potentiel de croissance ne l'expriment pas, cela se traduisant par une croissance moyenne faible mais homogène et par une survie importante (80\%). Dans les autres contextes de densités initiales effectives $\left(<9\right.$ ind. $\left./ \mathrm{m}^{2}\right)$, une croissance plus forte et/ou des écarts de croissance entre individus auraient provoqué l'apparition de comportements agressifs 
voire de cannibalisme se traduisant globalement par une croissance moyenne forte, mais hétérogène et par une survie faible. Ce phénomène a été souvent observé en aquaculture, notamment chez l'anguille dès que le poids moyen des plus gros individus dans un bassin dépasse 1,5 fois celui des plus petits (KNIGHTS, 1987). Le comportement grégaire initial des individus pourrait donc persister tant que des différences significatives de gabarit n'apparaissent pas au sein du groupe. La croissance de quelques individus au-delà d'un rapport d'environ 1,5 fois le poids des plus petits individus se traduirait alors par l'apparition d'actes agressifs voire de cannibalisme. Ce type de bascule comportementale est bien connu au cours du développement des alevins de brochet par exemple (BALVAY, 1983).

Le frein de croissance exercé sur les individus à bon potentiel dans les bassins à forte densité initiale pourrait venir d'une capacité trophique limitée par rapport à la densité de mise en charge en civelles. On peut également évoquer une cause sociale ou comportementale: comportements plus grégaires en forte densité, appropriation plus aisée de territoires en faible densité, comme cela a parfois été observé (LIGHTER, 1977 ; CARR et al., 1987). L'effet positif de la densité sur la démographie ou effet «Allee » (STEPHENS et al., 1999) est intéressant à noter. Dans le cas présent, il s'agirait d'un effet «Allee » « dû à l'augmentation de la capacité du milieu liée au maintien prolongé de la grégarité des individus au dessus d'un certain seuil de densité (par opposition à une territorialité en dessous de ce seuil).

\section{Dispersion des civelles}

Dans les plans d'eau, les civelles avaient l'opportunité d'une reprise alimentaire naturelle et/ou d'une émigration. Divers essais sur 5 mois en plans d'eau clos avaient révélé une croissance nettement densité dépendante pour des niveaux de densité bien inférieurs à ceux utilisés dans cette expérimentation (BELPAIRE et al., 1989, 1992 ; KLEIN BRETELER, 1992 ; RIGAUD et MASSE, 2001). On pouvait donc supposer qu'ayant la possibilité de sortir des plans d'eau, elles le feraient massivement notamment dans les contextes de forte densité initiale (9 à 12 ind./ $\mathrm{m}^{2}$ ). Or, les taux de sortie sont apparus modérés, concentrés dans le temps (sur la première période suivant la mise à l'eau) et liés de manière significative non pas à la densité initiale mais à la présence d'herbiers. Ces résultats peuvent être interprétés en se référant à l'hypothèse de FEUNTEUN et al. (2003) quant à l'existence de différents contingents. Dans les bassins à bonne capacité d'accueil (présence d'herbiers) les individus sortis du système pourraient ainsi être assimilés aux "pionniers « (ceux-ci constitueraient alors 7 à $15 \%$ du stock initial). Dans les bassins à faible capacité d'accueil (absence d'herbiers) les individus qui sont restés malgré tout pourraient être des »fondateurs « (représentant l'essentiel des civelles transférées soit 65 à $80 \%$ ). Les individus sortis lors des premiers jours sont apparus de taille et de poids moyens supérieurs à la moyenne du lot initial. L'autre hypothèse de travail propose l'existence de moments clés (VAN BAALEN et HOCHBERG, 2001). Le déversement a pu créer l'un de ces moments qui s'est traduit sur un temps assez court par un choix (rester ou partir) lié d'une part au gabarit des civelles et d'autre part à la capacité d'accueil du bassin concerné (présence d'herbiers). Le fait que les migrants possèdent des réserves ou des capacités physiques supérieures aux sédentaires a déjà été notée sur d'autres groupes d'animaux (CLOBERT et al., 2001), et a été observé chez la civelle en milieu expérimental (BARDONNET et al., 2003). Les expériences en bac montrent aussi que dans les premiers jours qui suivent la mise en place, les individus utilisent moins les abris. Ce comportement avait été interprété comme une phase exploratoire suivant la mise à l'eau. Ainsi, la mise en place des civelles dans un milieu nouveau (qu'il s'agisse des petits plans d'eau ou des bacs), semble être suivie par une phase de «non stabilité ». Au cours de cette phase, une partie des civelles exprime un niveau d'activité important qui se traduit par des sorties en bassin, ou par une proportion de civelles en nage ou sur le fond plus importante en bac. II est donc probable que les émigrations suivant la mise en place 
observées en bassin soient directement liées au transfert. II n'en reste pas moins que ce sont les plus petits individus qui restent et que la capacité d'accueil en abris joue un rôle important. Ce rôle a pu être démontré en plan d'eau, mais pas en bac, sans doute parce que l'effet «abri » joue en terme de présence/absence, et non en termes de densité.

On notera enfin, dans les 2 contextes expérimentaux, l'existence d'une phase longue de stabilité d' 1 mois sans émigration en plans d'eau et de 1 mois et demi en bac sans actes agressifs. Ce n'est qu'en toute fin de suivi que quelques rares sorties au regard du stock en place ont été de nouveau observées dans 5 des 9 bassins, différentes causes pouvant être évoquées. La fuite de plans d'eau où s'exerce une importante compétition en est une. On remarque d'ailleurs que les taux de sortie en fin de suivi par rapport aux stocks en place sont les plus importants non pas dans les bassins à fortes densités finales, mais dans les bassins à forte croissance hétérogène. On peut également évoquer des facteurs environnementaux externes ayant provoqué une réaction des individus, mais l'absence de phénomènes dans 4 des 9 bassins ne plaide pas vraiment pour une telle hypothèse. Les résultats des expériences sont cohérents avec ce qui est observé en bacs. Après 6 semaines de forte grégarité, les individus se dispersent dans le milieu et des actes agressifs apparaissent. KNIGHTS (1987) a étudié les relations agonistiques chez Anguilla anguilla. Les comportements agonistiques étaient importants avec une moyenne de 2,7 agressions par individu et par 5 minutes Cependant, il travaillait à des densités d'élevage très élevées (1 000 à 6000 ind./m²) et chez des individus plus âgés $(0,8$ à $1 \mathrm{~g}$ ). GLOVA et JELLYMAN (2000) ont travaillé à des densités beaucoup plus comparables (40 ind. $/ \mathrm{m}^{2}$ ) chez les juvéniles d'Anguilla australis et $A$. dieffenbachii. Ils observent de l'agressivité mais à des taux beaucoup plus faibles que KNIGHT (op. cited). A notre connaissance le comportement agressif chez la civelle n'a pas été rapporté en dehors de conditions de pisciculture. Leur apparition dans les bacs pourrait être le fait de l'atteinte d'un seuil en termes de différence de gabarit comme suggéré par les expériences en bassins.

\section{CONCLUSION}

On retiendra surtout l'existence d'une longue phase (4-6 semaines) sans changements comportementaux appréciables (en termes de déplacement ou de relations inter-individuelles), surprenante pour un stade biologique souvent associé à un comportement de déplacement et de migration. L'existence de cette phase est cohérente avec l'hypothèse d'une colonisation se déroulant par étapes. Ceci rappelle le schéma de migration par étapes récemment mis en évidence chez l'autre stade migrant, l'anguille argentée (DURIF, 2002)

Par ailleurs, les résultats suggèrent l'existence de pressions de compétition interindividuelle en lien avec l'hétérogénéité de croissance qui pourrait influer sur la reprise des déplacements après une phase de stabulation plus ou moins longue.

\section{REMERCIEMENTS}

Ce projet a été retenu par le Grisam (Groupe d'Intérêt Scientifique sur les Poissons Amphihalins) et soutenu financièrement par le MEDD via la Diren Aquitaine. Les auteurs tiennent à remercier Maël Hervouet et Emmanuel Huchet pour leur contribution au déroulement des expérimentations ainsi que Jacques Rives pour sa participation. Remerciements au Conservatoire du Littoral et des Rivages lacustres pour la mise à disposition des plans d'eau expérimentaux sur le Domaine de Certes à Audenge (Gironde). Nous remercions également deux relecteurs anonymes dont les remarques ont largement contribué à l'amélioration du document initial. 


\section{BIBLIOGRAPHIE}

BALVAY G., 1983. L'alimentation naturelle des alevins de brochet (Esox lucius) durant leur premier mois de vie. In: Le brochet: gestion dans le milieu naturel et élevage, BILLARD R. (Ed.), Inra Publi., Paris, 179-198.

BARDONNET A., DASSE S., PARADE M., HELAND M., 2003. Influence de l'alternance jour/nuit sur les déplacements de civelles en fluvarium. Bull. Fr. Pêche Piscic. 368, 9-20.

BELPAIRE C., VAN VLASSELAER L., PODOOR N., OLLEVIER F., 1989. Results of pond culture experiments with glass eel of the European eel (A. anguilla), EIFAC, Working Group on Eel, Porto, 14 p.

BELPAIRE C., VAN DRIESSCHE H., GAO F.Y., OLLEVIER F., 1992. Food and feeding activity of glass eel Anguilla anguilla (L.) stocked in earthen ponds. Irish Fisheries Investigations Series A (Freshwater), 36, 43-54.

BURNHAM K.P., ANDERSON D.R., 1998. Model selection and multimodel inference : a practical information-theoretic approach. Springer-Verlag, New York.

CARR M.J., CARR J.E., JOHNSON S.R., 1987. Seasonal changes in territoriality and frequency of agonistic behavior in two densities of juvenile brown bullhead, Ictalurus nebulosus. Environmental Biology of Fishes, 19, 175-181.

CLOBERT J., DANCHIN E., DHONDT A.A., NICHOLS J.D., 2001. Dispersal. First edition, Oxford University Press, Oxford.

CREUTZBERG F., 1961. On the orientation of the migrating elvers (A. vulgaris) in a tidal area. Netherland Journal of Sea Research, 1, 257-338.

DE LEO G.A., GATTO M., 1996. Trends in vital rates of the European eel : evidence for density dependance. Ecological Applications, 6(4), 1281-1294.

DOU S.-Z., TSUKAMOTO K., 2003. Observations of the nocturnal activity and feeding behavior of the Anguilla japonica glass eels under laboratory conditions. Environmental Biology of Fishes, 67, 389-396.

DURIF C., ELIE P., GOSSET C., RIVES J., TRAVADE F., 2003. Behavioral study of downstream migrating eels by radio-telemetry at a small hydroelectric power plant. In : Biology, Management, and Protection of Catadromous Eels, DIXON D.A. (Ed.) American Fisheries Society, Symposium 33. Bethesda, Maryland, 345-356.

ELIE P., LECOMTE-FINIGER R., CANTRELLE I., CHARLON N., 1982. Définition des limites des différents stades pigmentaires durant la phase civelle d'Anguilla anguilla $\mathrm{L}$. (poisson téléostéen anguilliforme). Vie et Milieu, 32 (3), 149-157.

ELIE P., ROCHARD E., 1994. Migration des civelles d'anguilles (Anguilla anguilla) dans les estuaires, modalités du phénomène et caractéristiques des individus. Analyse de la littérature. Bull. Fr. Pêche Pisc., 335, 81-98.

FEUNTEUN E., LAFFAILLE P., ROBINET T., BRIAND C., BAISEZ A., OLIVIER J.M., ACOU A., 2003. A review of upstream migration and movements in inland waters by anguillid eels : towards a general theory. In : Eel Biology, AIDA K., TSUKAMOTO K., YAMAUCHI K. (Eds), 191-213

FONTAINE M., CALLAMAND O., 1941. Sur l'hydrotropisme des civelles. Bull. Inst. Océan., 811, 1-6.

GASCUEL D., 1986. Flow-carried and active swimming migration of the glass-eel (A. anguilla) in the tidal area of a small estuary on the French Atlantic coast. Helgolander Meeresunters, 40, 321-326. 
GERDEAUX D., 1987. Revue des méthodes d'estimation de l'effectif d'une population par pêches successives avec retrait. Programme d'estimation d'effectifs par la méthode de Carle et Strub. Bull. Fr. Pêche Pisc., 304, 13-21.

GLOVA G.J., 2001. Effects of the presence of subadult longfinned eels (Anguilla dieffenbachii) on cover preferences of juvenile eels (Anguilla spp.) in replicate channels. New Zealand Journal of Marine and Freshwater Research, 35, 221-233.

GLOVA G.J., 2002. Density effects of the shortfinned eel (Anguilla australis) cover preferences in replicate channels. New Zealand Journal of Marine and Freshwater Research, 36, 483-490.

GLOVA G.J., JELLYMAN D.J., 2000. Size-related differences in diel activity of two species of juvenile eel (Anguilla) in a laboratory stream. Ecology of Freshwater Fish, 9, 210218.

IBBOTSON A., SMITH J., SCARLETT P., APRAHAMIAN M., 2002. Colonisation of freshwater habitats by the European eel Anguilla anguilla. Freshwater Biology, 47 (9), 1696-1706.

JELLYMAN D.J., 1997. Variability in growth rates of freshwater eels (Anguilla spp.) in New Zealand Ecology of Freshwater Fish, 6, 108-115.

JELLYMAN D.J., CHISNALL B.L., 1999. Habitat preferences of shortfinned eels (Anguilla australis), in two New Zealand lowland lakes. New Zealand Journal of Marine and Freshwater Research, 33, 233-248.

KLEIN BRETELER J.G.P., 1992. Effect of provenance and density on growth and survival of glass eels Anguilla anguilla (L.) in mesocosm experiments. Irish Fisheries Investigations Series A (Freshwater), 36, 15-22.

KNIGHTS B., 1987. Agonistic behaviour and growth in the European eel, A. anguilla L, in relation to warm-water aquaculture. Journal of Fish Biology, 31, 265-276.

LAFFAILLE P., BAISEZ A., RIGAUD C., FEUNTEUN E., 2004. Habitat preferences of different eel size classes in a reclaimed marsh. A contribution to species and ecosystem conservation. Wetlands, 24(3), 642-651.

LECOMTE-FINIGER R., PRODON R., 1979. Etude expérimentale du comportement fouisseur de la civelle: le choix d'un substrat. C.R. Acad. Sci., Paris, 289, 741743.

LIGHTER F.J., 1976. The social use of space in the Hawaiian ghost crab, Ocypode ceratophtalmus. Pacific Science, 30, 211-212.

NAISMITH I.A., KNIGHTS B., 1993. The distribution, density and growth of the eel $(A$. anguilla) in the freshwater catchment of the River Thames. Journal of Fish Biology, 37, 217-226.

OLIVEIRA K., MCCLEAVE J.D., WIPPELHAUSER G.S., 2001. Regional variation and the effect of lake/river area on sex distribution of American eels. Journal of Fish Biology, 58(4), 943-952.

PARSONS J., VICKERS K.U., WARDEN Y., 1977. Relationship between elver recruitment and changes in the sex ratio of silver eels Anguilla anguilla L. migrating from Lough Neagh, Northern Ireland. Journal of Fish Biology, 10, 211-229.

RIGAUD C., MASSÉ J., 2001. Démarche agro-piscicole menée sur le marais de la Côte neuve à Bourcefranc (Charente-Maritime). Analyse des alevinages d'anguilles en fossés expérimentaux entre 1998 et 2000. Etude $n^{\circ}$ 64, Cemagref Bordeaux, $31 \mathrm{p}$. 
SILBERSCHNEIDER V., PEASE B.C., BOOTH D.J., 2004. Estuarine habitat preferences of Anguilla australis and $A$. reinhadtii glass eels inferred from laboratory experiments. Environmental Biology of Fishes, 71, 395-402.

SMOGOR R.A., ANGERMEIER P.L., GAYLORD C.K., 1995. Distribution and abundance of American eels in Virginia streams: Tests of null models across spatial scales. Transactions of the American Fisheries Society, 124, 789-803.

STEPHENS P.A, SUTHERLAND W.J., FRECKLETON R.P., 1999. What is the Allee effect ? Oikos, 185-190.

TESCH F.-W., 1977. The eel : Biology and management of anguillid eels. GREENWOOD P.H. (Ed.), Chapman and Hall.

TZENG W.N, SEVERIN K.P., WICKSTROM H., 1997. Use of otolith microchemistry to investigate the environmental history of European eel (A. anguilla). Mar. Ecol. Prog. Ser., 149, 73-81.

VAN BAALEN M., HOCHBERG M.E., 2001. Dispersal in antagonistic interactions. In : Dispersal, CLOBERT J., DANCHIN E., DHONDT A.A., NICHOLS J.D (Eds), 299310.

VOLLESTAD L.A., JONSSON B., 1986. Life history characteristics of the European eel, Anguilla anguilla in the Imsa river, Norway. Transactions of the American Fisheries Society, 115, 864-871.

VOLLESTAD L.A., JONSSON B., 1988. A 13-year study of the populations dynamics and growth of the European eel, $A$. anguilla in a Norvegian river : evidence for densitydependent mortality, and development of a model for predicting year. Journal of Animal Ecology, 57, 983-997.

WHITE E.M., KNIGHTS B., 1997. Environmental factors affecting migration of the European eel in the rivers Severn and Avon, England. Journal of Fish Biology, 50, 1104-1116. 
